\title{
Development of a diagnostic device to detect different Pseudomonas aeruginosa phenotypes in medically relevant contexts
}

\author{
Andrew C. Ward, Nicholas P. Tucker, Patricia Connolly
}

\begin{abstract}
Pseudomonas aeruginosa, widely present in the environment, is well known for its ability to cause infection in immune compromised individuals. For example, $P$. aeruginosa is the leading cause of morbidity and mortality in patients with cystic fibrosis (CF). Here, we describe how Electrochemical Impedance Spectroscopy (EIS) can be used to detect the presence of four different strains of $P$. aeruginosa. Using a low cost, screen printed carbon electrode significant changes can be seen in impedance data in the presence of $P$. aeruginos $a$ after 24 hours. Furthermore, through the use of a normalization technique whereby the phase angle of the impedance (a commonly used parameter) is divided by a starting measurement, it is possible to identify differences between a non-mucoid and mucoid strain of $P$. aeruginosa. Sensors based upon the techniques described here could be used in a number of healthcare scenarios, where there is a need for low cost, real time detection of $P$. aeruginosa, such as CF.
\end{abstract}

\section{INTRODUCTION}

Pseudomonas aeruginosa is a medically important gram negative bacterium responsible for causing infection in immune compromised individuals. Chronic lung infections due to $P$. aeruginosa are the leading cause of morbidity and mortality in patients with cystic fibrosis (CF)[1]. Burns are also particularly vulnerable to colonization by $P$. aeruginosa [2] and there are a number of other groups who may succumb to the infection including pre-term babies [3]. $P$. aeruginosa infections have also been associated with blood stream infections (BSI). BSIs have a large economic burden, with the cost of a treatment estimated to be $\$ 40,000$ per patient [4].

In $\mathrm{CF}$ patients, initial virulent strains of $P$. aeruginosa become rapidly host adapted, forming biofilms which make them resistant to eradication either by the host immune system or by antibiotics [5]. Virulent strains of $P$. aeruginosa are generally motile and planktonic whereas host adapted strains are sessile and produce large quantities of alginate. The virulent strains are often described as "nonmucoid", in contrast to their alginate producing, "mucoid" counterparts. Early detection of the non-mucoid strain before the bacteria become host adapted may make it possible to eradicate $P$. aeruginosa, thus preventing chronic

This work has been funded by the Engineering and Physical Sciences Research Council (www.epsrc.ac.uk, grant number: EP/F50036X/1) as part of an Engineering Doctorate.

A. C. Ward is with the University of Strathclyde, Wolfson Building, 106 Rottenrow, Glasgow, G4 0NW, UK. (andrew.c.ward@strath.ac.uk).

N. P. Tucker is with the University of Strathclyde (nick.tucker@strath.ac.uk).

P. Connolly is with the University of Strathclyde (corresponding author. patricia.connolly@strath.ac.uk. Tel. 44141548 3034) colonisation [6]. A technique that enables real time detection of these two strains in a sample could therefore be clinically useful.

Electrochemical Impedance Spectroscopy (EIS) has been explored previously as a potential technique for monitoring or characterising $P$. aeruginosa [7]-[9]. Whilst much research has been carried out using EIS, the dependence upon labels (such as antibodies or DNA oligonucleotides) or expensive electrode materials and manufacturing techniques make application in low cost point of care devices challenging. In this study, we have combined the use of low cost, disposable screen printed carbon electrodes with EIS in order to detect the presence of $P$. aeruginosa. Previously, we have shown how $P$. aeruginosa is detectable in a polymicrobial competition model using a screen printed carbon electrode [10]. In other work, it has also been shown that screen printed silver chloride electrodes can be used to detect $S$. aureus in a microbial broth [11]. Screen printing has been applied previously as a basis for low cost sensors, such as the detection of moisture within a chronic wound [12], [13]. The technique has been granted an EU patent for its use in cell and bacterial recognition and patents owned by the University of Strathclyde are pending worldwide [14].

The impedance characteristics of an electrode-electrolyte interface are governed by a number of processes [15]. The concentration of charged molecules and solutes within the electrode affect the capacitance, the presence of redox active compounds can affect the charge transfer capabilities of the interface. The diffusion of any redox active compounds will also influence the rate of charge transfer, along with the concentration of these compounds within the media. The conductivity of electrolyte between the electrodes also has an impact upon the measured impedance at high frequencies. We hypothesize that it is possible to detect the presence of microorganisms by looking across the impedance spectrum for specific changes to the characteristics of the interface. In this study, we focus upon the impedance signature caused by $P$. aeruginosa and demonstrate differences in the impedance between non-mucoid and mucoid strains.

\section{METHODS}

\section{A. Strains and Media}

All experiments were performed in Lysogeny Broth (LB) media (described previously [10]). Two non-mucoid/mucoid strain pairs of $P$. aeruginosa were tested: J1385/J1532 and $\mathrm{C} 1446 / \mathrm{C} 1433$. These are clinical isolates from CF sputum and were kindly provided by the UK Cystic Fibrosis Microbiology Consortium at the University of Edinburgh 
[16]. J1385 and C1426 are non-mucoid strains and J1532 and $\mathrm{C} 1433$ are mucoid strains. All measurements were replicated with four different samples for each strain. Final concentrations of $P$. aeruginosa at the end of the experiment were determined using the drop plate method [17], as described previously [10].

\section{B. Electrode Manufacture and Normalization}

Carbon electrodes were screen printed onto acetate, with a section of $15 \mathrm{ml}$ centrifuge tube mounted on top of them [10]. The impedance measurements resulting from the experiment were normalized in order to distinguish differences in the impedance caused by $P$. aeruginosa [14]. This was achieved using the following equation:

$$
I P N_{t=n}=\frac{I P A_{t=n}}{I P A_{t=0}}
$$

Where $I P N_{t=n}$ is the normalized impedance parameter of interest (i.e. reactance, resistance, impedance modulus or phase) and $I P A_{t}$ is the absolute (as measured) impedance parameter.

\section{Impedance Measurement and Growth Experiments}

Electrodes chambers were sterilized in $70 \% \mathrm{v} / \mathrm{v}$ ethanol and then inoculated in replicates of four with $10 \mu \mathrm{l}$ of bacterial broth containing approximately $3.5 \times 10^{9}$ colony forming units (CFU) per milliliter of the strain of interest. Four electrodes were filled with sterile media as negative controls. Electrodes were incubated for 72 hours at $37^{\circ} \mathrm{C}$ in air. In order to prevent the samples drying out during the incubation period, the humidity was increased through the use of wet paper towel placed around the chambers. A plastic container was then placed over the electrodes to allow the humidity to increase. Impedance measurements were taken with a Solartron 1260 impedance analyzer with an AC potential of $200 \mathrm{mV}_{\mathrm{rms}}$ from $1 \mathrm{MHz}$ to $0.1 \mathrm{~Hz}$ at the start of the experiment and then once every 24 hours.

\section{Statistical analysis}

The Mann-Whitney test was used to compare the results against the negative control. Minitab was used for the statistical analysis. Statistical significance is defined here as $P \leq 0.05$.

TABLE 1. MEAN CHANGE IN IMPEDANCE MODULUS AT LOW FREQUENCY FOR EACH STRAIN. VALUES MARKED * ARE SIGNIFICANTLY DIFFERENT TO THE NEGATIVE CONTROL.

\begin{tabular}{|l|c|c|c|c|}
\hline \multirow{2}{*}{ Strain } & \multicolumn{4}{|c|}{ Impedance Modulus at 0.1 Hz (Ohms) } \\
\cline { 2 - 5 } & 0 Hours & 24 Hours & 50 Hours & 72 Hours \\
\hline $\mathrm{Neg}(n=4)$ & $4.51 \times 10^{6}$ & $4.39 \times 10^{6}$ & $4.20 \times 10^{6}$ & $4.09 \times 10^{6}$ \\
\hline $\mathrm{C} 1426(n=4)$ & $5.27 \times 10^{6}$ & $1.13 \times 10^{6} *$ & $5.28 \times 10^{5} *$ & $3.04 \times 10^{5} *$ \\
\hline $\mathrm{C} 1433(n=4)$ & $4.73 \times 10^{6}$ & $3.03 \times 10^{5} *$ & $2.00 \times 10^{5} *$ & $2.47 \times 10^{5} *$ \\
\hline $\mathrm{J} 1385(n=4)$ & $4.98 \times 10^{6}$ & $7.33 \times 10^{5} *$ & $2.95 \times 10^{5} *$ & $2.10 \times 10^{5} *$ \\
\hline $\mathrm{J} 1532(n=4)$ & $5.58 \times 10^{6}$ & $5.35 \times 10^{5} *$ & $2.87 \times 10^{5} *$ & $2.14 \times 10^{5} *$ \\
\hline
\end{tabular}

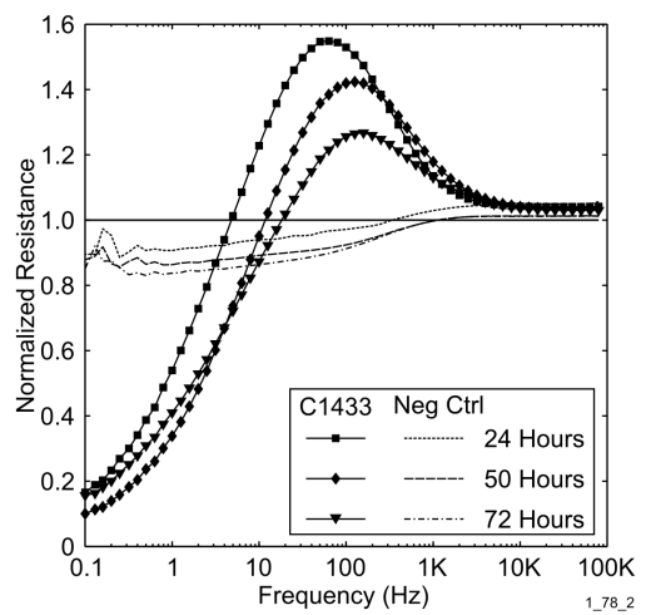

Figure 1. Example of normalized resistance peaks present in the mucoid strain C1433 over the course of the experiment. This peak was typical of both non-mucoid and mucoid strains. Data represents the mean from 4 measurements, error bars omitted for clarity.

\section{RESULTS}

\section{A. Growth of Bacteria during Experiment}

After 24 hours, all strains of $P$. aeruginosa produced a change in the impedance resistance, reactance and associated normalized impedance parameters contrasted to the negative controls. Characteristic impedance peaks occurred in the normalized resistance data after 24 hours (figure 1). These are similar to those observed previously, with a different $P$. aeruginosa strain, PA14 [10].

A significant drop in the impedance modulus at low frequency could be seen with each of the strains contrasted to the negative control, indicating a decrease in the impedance of the sensor as a consequence of the growth of $P$. aeruginosa (table 1).

\section{B. Non-mucoid vs. Mucoid Impedance Changes}

The impedance signatures from each of the nonmucoid/mucoid pairs were compared to determine if it is possible to detect any difference between a virulent strain of $P$. aeruginosa and a host adapted strain. Comparison of the non-mucoid and mucoid mean data shows that a clear peak and trough pair are present in the data from the non-mucoid strain around $100 \mathrm{~Hz}$, with a trough present between $1 \mathrm{KHz}$ and $2.5 \mathrm{KHz}$ (figure 2A). In contrast, only one peak is observable at $100 \mathrm{~Hz}$ for the mucoid strain, seen in the data for $\mathrm{C} 1433$ at 72 hours (figure $2 \mathrm{~B}$ ). Troughs are still visible in some of the data from the mucoid strains, as seen in mean data for J1532 and C1433 (figure 2B).

When the normalized phase angle at $100 \mathrm{~Hz}$ is analyzed, a clear difference is observable between the non-mucoid strains and mucoid strains. Specifically, it can be seen that the non-mucoid strains have a higher normalized phase angle than the mucoid strains (figure $3 \mathrm{~A}$ and figure $3 \mathrm{~B}$ ). Furthermore, when the data from the two non-mucoid strains and the data from the two mucoid strains are pooled, there is a significant different in the normalized phase angle at 24 
hours and 48 hours $(P=0.0014$ and $P=0.0039$ respectively), although this difference disappears at later time points.

\section{Post experiment cell density}

Colony counting was carried out at the end of the experiment in order to determine the $P$. aeruginosa cell density within each of the electrode chambers. A similar concentration of cells was found across all strains (table 2), with the exception of $\mathrm{J} 1532$, where a slightly lower concentration was counted. This could have been related to the viscous media present at the end of the experiment which made it difficult to homogenize the sample and perform serial dilutions equally, thus possibly leading to an underestimate of the number of viable cells present.

\section{DISCUSSION}

These results demonstrate that it is possible to detect the presence of four different strains of $P$. aeruginosa, all of which were clinical isolates originally from $\mathrm{CF}$ sputum. The carbon electrode has a high starting impedance, which could mask some of the impedance changes or reduce the sensitivity of the electrode. The drop in the modulus of impedance for electrodes inoculated with $P$. aeruginosa was found previously to be caused at least in part by a set of electroactive compounds produced by $P$. aeruginosa called phenazines [10]. These compounds fundamentally change the electrode-electrolyte interface to facilitate electron transfer that isn't possible in the control chambers. A similar effect could be occurring here, suggesting that clinical isolates could be detected through the same mechanism.

Whilst the experimental arrangement described here is far from the same as the composition of a clinical sample, it is interesting to note that slight but significant differences in impedance characteristics have been found between the nonmucoid and mucoid strains. The similarity between the bacterial cell densities of each strain at the end of the experiment suggest that the changes are not related to a difference in cell concentration. Furthermore, it is possible that the peaks observed in the normalized phase angle are caused by phenotype differences between the non-mucoid and mucoid strains. Specifically, the changes could be related to different concentrations of phenazine compounds produced by each of the strains.

TABLE 2. FINAL $P$. AERUGINOSA CELL DENSITIES DETERMINED THROUGH DROP PLATE COLONY COUNTING

\begin{tabular}{|l|l|c|c|}
\hline \multirow{2}{*}{ Strain } & \multicolumn{3}{|c|}{$\begin{array}{c}\text { P. aeruginosa cell density at 72 Hours } \\
\text { (CFU/ml) }\end{array}$} \\
\cline { 2 - 4 } & \multicolumn{1}{|c|}{ Mean } & Min & \multicolumn{1}{c|}{ Max } \\
\hline $\mathrm{C} 1426(n=4)$ & $5.96 \times 10^{9}$ & $5.20 \times 10^{8}$ & $1.65 \times 10^{10}$ \\
\hline $\mathrm{C} 1433(n=4)$ & $6.80 \times 10^{9}$ & $1.68 \times 10^{9}$ & $2.00 \times 10^{10}$ \\
\hline $\mathrm{J} 1385(n=4)$ & $5.28 \times 10^{9}$ & $1.55 \times 10^{8}$ & $1.27 \times 10^{10}$ \\
\hline $\mathrm{J} 1532(n=4)$ & $4.60 \times 10^{8}$ & $7.00 \times 10^{6}$ & $1.63 \times 10^{9}$ \\
\hline
\end{tabular}
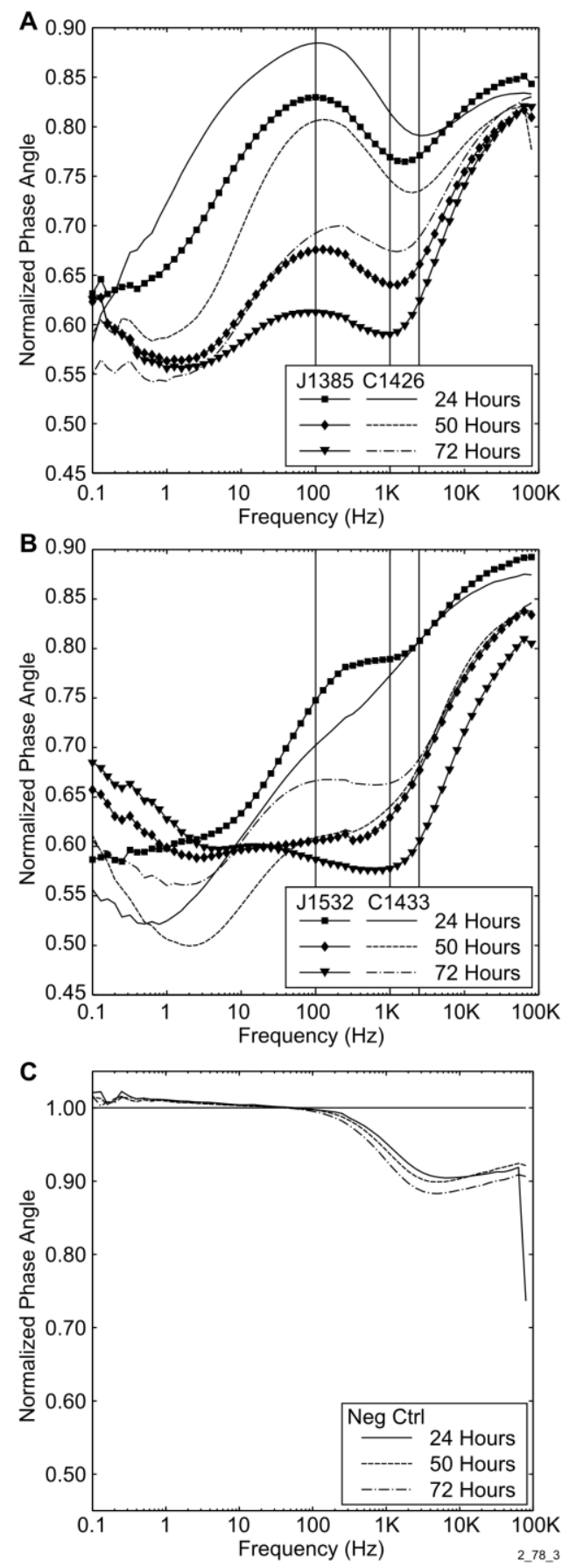

Figure 2. Comparison of the mean $(n=4)$ normalised phase angle between non-mucoid and mucoid pairs across the frequency spectrum. (A) non-mucoid strains J1385 and C1426, showing a clear peak at approximately $100 \mathrm{~Hz}$ and a trough between $1 \mathrm{KHz}$ and $2.5 \mathrm{KHz}$ across all data points (black vertical lines). (B) Mucoid strains J1532 and C1433, showing that only one slight peak exists at 72 hours in the data from C1433. (C) Negative control showing the stability of the electrode over the course of the experiment. Results represent the mean from 4 replicates, error bars not included for clarity.

Alternatively, the differences could be caused by other phenotypic changes in the bacteria, such as an interaction between alginate produced by the mucoid strain and the electrode-electrolyte interface. Further investigation is required to identify the underlying cause for the impedance change between the non-mucoid and mucoid strains. 

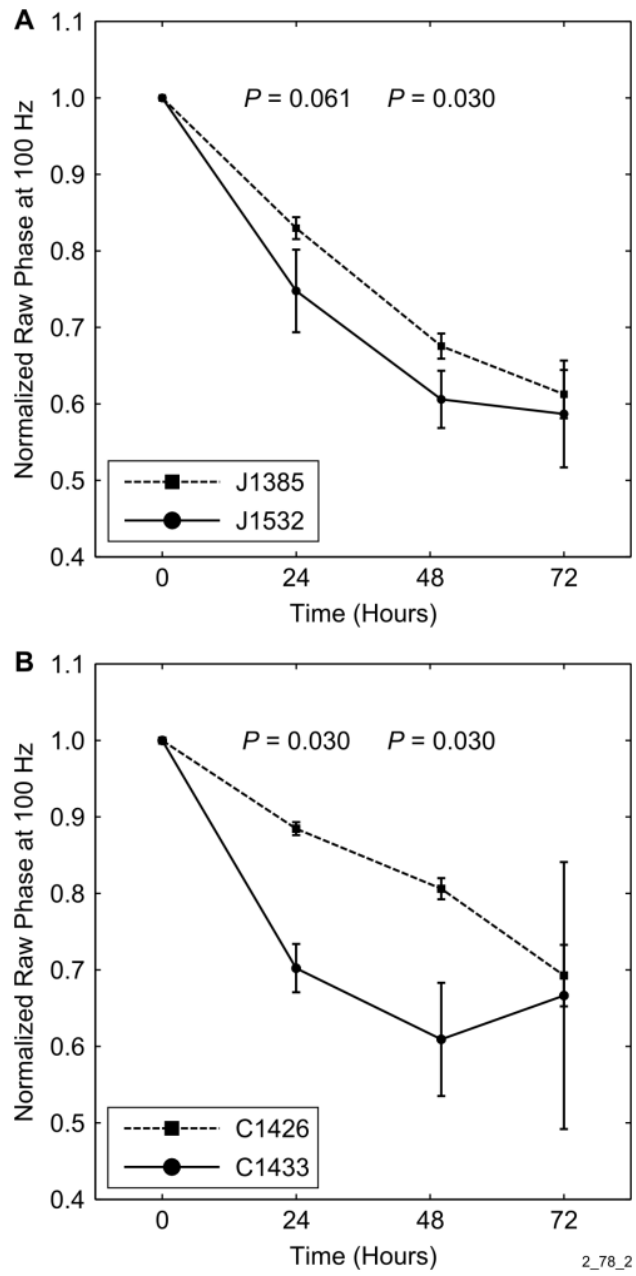

Figure 3. Comparison of the normalized phase angle between nonmucoid and mucoid pairs at $100 \mathrm{~Hz}$. (A) J1385/J1532 pair and (B) C1426/C1433 pair. Error bars represent $+/-1 \mathrm{SD}, n=4$.

The measurement voltage of $200 \mathrm{mV}_{\text {rms }}$ used for the impedance measurement is greater than the magnitude over which the electrochemical cell behaves in a linear manner [15], [18]. However, at smaller measurement voltages, it was found that the low frequency (i.e. less than $1 \mathrm{~Hz}$ ) impedance data were noisy making these potentials impractical for analysis (data not shown). This was probably caused by the limitations of the equipment given the high electrode impedance.

\section{CONCLUSION}

The experiments described here show how EIS, combined with a low cost screen printed sensor could be used to detect the presence of $P$. aeruginosa. The normalization procedure described can be used as a powerful technique to elucidate differences between $P$. aeruginosa strains, under laboratory conditions. Future development may lead to a sensor that is capable to detecting phenotypic changes in $P$. aeruginosa in clinically important samples, such as sputum from CF patients.

\section{REFERENCES}

[1] G. Döring, P. Flume, H. Heijerman, J. S. Elborn, and for the Consensus Study Group, "Treatment of lung infection in patients with cystic fibrosis: Current and future strategies," J. Cyst. Fibros., vol. 11, no. 6, pp. 461-479, Dec. 2012.

[2] L. K. Branski, A. Al-Mousawi, H. Rivero, M. G. Jeschke, A. P. Sanford, and D. N. Herndon, "Emerging infections in burns," Surg. Infect., vol. 10, no. 5, pp. 389-397, Oct. 2009.

[3] J. M. C. Jefferies, T. Cooper, T. Yam, and S. C. Clarke, "Pseudomonas aeruginosa outbreaks in the neonatal intensive care unit - a systematic review of risk factors and environmental sources," J. Med. Microbiol., vol. 61, no. Pt_8, pp. 1052-1061, Jun. 2012.

[4] A. M. Elward, C. S. Hollenbeak, D. K. Warren, and V. J. Fraser, "Attributable Cost of Nosocomial Primary Bloodstream Infection in Pediatric Intensive Care Unit Patients," PEDIATRICS, vol. 115, no. 4, pp. 868-872, Apr. 2005.

[5] D. J. Hassett, M. D. Sutton, M. J. Schurr, A. B. Herr, C. C. Caldwell, and J. O. Matu, "Pseudomonas aeruginosa hypoxic or anaerobic biofilm infections within cystic fibrosis airways," Trends Microbiol., vol. 17, no. 3, pp. 130-138, Mar. 2009.

[6] P. Schelstraete, F. Haerynck, Van daele Sabine, S. Deseyne, and F. De Baets, "Eradication therapy for Pseudomonas aeruginosa colonization episodes in cystic fibrosis patients not chronically colonized by P. aeruginosa," J. Cyst. Fibros., vol. 12, no. 1, pp. 18, Jan. 2013.

[7] A. Dheilly, I. Linossier, A. Darchen, D. Hadjiev, C. Corbel, and V. Alonso, "Monitoring of microbial adhesion and biofilm growth using electrochemical impedancemetry," Appl. Microbiol. Biotechnol., vol. 79, pp. 157-164, Mar. 2008.

[8] T. Kim, J. Kang, J. H. Lee, and J. Yoon, "Influence of attached bacteria and biofilm on double-layer capacitance during biofilm monitoring by electrochemical impedance spectroscopy," Water Res., vol. 45, pp. 4615-4622, 2011.

[9] S. Kim, G. Yu, T. Kim, K. Shin, and J. Yoon, "Rapid bacterial detection with an interdigitated array electrode by electrochemical impedance spectroscopy," Electrochimica Acta, vol. 82, pp. 126131, Nov. 2012.

[10] A. C. Ward, P. Connolly, and N. P. Tucker, "Pseudomonas aeruginosa Can Be Detected in a Polymicrobial Competition Model Using Impedance Spectroscopy with a Novel Biosensor," PLoS ONE, vol. 9, no. 3, p. e91732, Mar. 2014.

[11] M. J. Farrow, I. S. Hunter, and P. Connolly, "Developing a Real Time Sensing System to Monitor Bacteria in Wound Dressings," Biosensors, vol. 2, no. 4, pp. 171-188, May 2012.

[12] D. McColl, B. Cartlidge, and P. Connolly, "Real-time monitoring of moisture levels in wound dressings in vitro: An experimental study," Int. J. Surg., vol. 5, no. 5, pp. 316-322, 2007.

[13] D. McColl, M. MacDougall, L. Watret, and P. Connolly, "Monitoring moisture without disturbing the wound dressing," Wounds UK, vol. 5, no. 3, pp. 94-99, 2009.

[14] P. Connolly and L. Shedden, "A System and Method for Cell Characterisation. Patent application number: WO2009136157 (A3)," WO2009136157 (A3), 28-Jan-2010.

[15] J. R. Macdonald, Impedance spectroscopy : emphasizing solid materials and systems. New York: Wiley, 1987.

[16] L. Stewart, A. Ford, V. Sangal, J. Jeukens, B. Boyle, S. Caim, L. Crossman, P. A. Hoskisson, R. Levesque, and N. P. Tucker, "Draft genomes of twelve host adapted and environmental isolates of Pseudomonas aeruginosa and their position in the core genome phylogeny," Pathog. Dis., p. In press, 2013.

[17] B. Herigstad, M. Hamilton, and J. Heersink, "How to optimize the drop plate method for enumerating bacteria," J. Microbiol. Methods, vol. 44, no. 2, pp. 121-129, 2001.

[18] A. J. Bard and L. R. Faulkner, Electrochemical methods: fundamentals and applications, 2 edn. New York: Wiley, 2001. 